\title{
Color, Size and Shape Feature Extraction Techniques for Fruits: A Technical Review
}

\author{
Amish Patel \\ Assistant Professor \\ Uka Tarsadia University, \\ Bardoli, Gujarat
}

\author{
Puja Kadam \\ Assistant Professor \\ Uka Tarsadia University \\ Bardoli, Gujarat
}

\author{
Sapan Naik \\ Assistant Professor \\ Uka Tarsadia University \\ Bardoli, Gujarat
}

\begin{abstract}
Grading of agricultural produce especially the fruits and vegetables has become a perquisite of trading across borders. In India mostly fruit and vegetable growers grade the fruit manually. Manual grading was carried out by trained operators who considered a number of grading factors and fruit were separated according to their physical quality . Manually grading was costly and grading operation was affected due to shortage of labor in peak seasons. Human operations may be inconsistent, less efficient and time consuming. New trends in marketing as specified by World Trade Organization (WTO) demand high quality graded products. Farmers are looking forward to having an appropriate agricultural produce-grading machine in order to alleviate the labor shortage, save time and improve graded product's quality.. The need to be responsive to market demand places a greater emphasis on quality assessment, resulting in the greater need for improved and more accurate grading and sorting practices. Size variation in vegetables like potatoes, onions provided a base for grading them in different categories. Every vegetable producing country had made their own standards of different grades keeping in view the market requirements.
\end{abstract}

\section{Keywords}

Fruit grading, Color Feature Extraction, Shape Feature Extraction, Size Feature Extraction.

\section{INTRODUCTION}

India is an agricultural country, where about $70 \%$ of people spend their lives by agriculture. Farmers have wide range of fruits and vegetable crops to harvest. The quality and optimal production of crops highly depends upon scientific reasons [16]. The management of permanent fruit crops requires close monitoring especially for the managing of diseases that can affect fabrication considerably and consequently the post harvest life. The image processing can be highly applied on agricultural applications for various purposes like:

\section{To detect diseased leaf, stem, fruit and roots. \\ 2. To determine size $\&$ shape of fruits and plant. \\ 3. To estimate chlorophyll content of a plant. \\ 4. Measurement of plant leaf area. \\ 5. Weed detection}

The fruits sorting and grading are considered the most important steps of handling. Sorting and grading are major processing tasks associated with the production of freshmarket fruit, vegetable and crop types. Post harvest process of fruits and vegetables is considered as the most important process that leads to conserve the quality until reach to the consumers. Sorting is a separation based on a single measurable property of raw material units, while grading is "the assessment of the overall quality of a food using a number of attributes". Sorting of agricultural products is accomplished based on appearance (color and absence defects), texture, shape and sizes [17]. Manual sorting is based on conventional visual quality inspection performed by human operators, which is tedious, time-consuming, slow and non-consistent. It has become increasingly difficult to hire personnel who are adequately trained and willing to undertake the tedious task of inspection. A cost effective, consistent, superior speed and accurate sorting can be achieved with machine vision assisted sorting. Grading of fruits, vegetables and crops is a very important operation as it fetches high price to the grower and improves packaging, handling and brings an overall improvement in marketing system. They are generally graded on the basis of size and graded products are more welcome in export market. Grading could reduce handling losses during transportation. Grading based on size consists of divergent roller type principle having inclination, expanding pitch type, inclined vibrating plate and counter rotating roller having inclination type graders. Weight grading based on density and specific gravity of agricultural commodities

In this paper, we have given brief overview on how fruits and vegetables can be graded and sorted. In the second section shape feature extraction methods are discussed, in third section color feature extraction techniques are described, following size extraction methods in fourth section are conversed. In fifth section conclusion has been given after the review of different techniques based on size, shape and color feature extraction using image processing.

\section{SHAPE FEATURE EXTRACTION AND ALGORITHMS}

In [1] Hatou used a triangulation laser range finder for constructing the 3-D shape of tomato fruits as they traveled on a conveyor belt beneath the range finder or scanner. Initially, a reference or ideal tomato shape was built from measurements obtained using the same laser range scanner. The inspected shape of each fruit was compared with the reference shape, and the differences between the two shapes were used for classification. Using an intelligent classifier system which included neural networks and expert system this was performed. The authors originated that the grading results of their system were similar to those achieved by a skilled human inspector. The disadvantage was that the method was time-consuming - it took $5 \mathrm{~s}$ to classify each tomato.

Passive 3-D machine vision methods are recognized in the literature as shape or range from $\mathrm{X}$ techniques, where $\mathrm{X}$ represents different 3-D cues such as stereo, shading, silhouettes or occluding contours,

motion, contour or shape, shadows or darkness, texture, and fractal geometry. From one or more 2D images these 3D cues can be determined. [2] It is sufficient to say that surface orientation can be obtained from the range by taking 
derivatives to clarify the relationship between depth or range on one side and surface orientation on the other hand. With the exemption of stereovision, shape from $\mathrm{X}$ methods cannot be used to directly calculate absolute depth, only relative depths and the shape from $\mathrm{X}$ methods usually require assumptions of certain regularities. Conclusively, due to these reasons they are considered as ill-posed problems.

Equatorial or transversal diameter and the polar diameter or length are the typical axes used for fruit size and simple shape determination. These involves vernire caliper as a reference method for measuring these diameters on small and medium sized fruits. Owing to the odd shapes of horticultural produce, shape and dimension or size are 3-D values; that is, the same measurement on the same fruit often varies in different planes around the central axis of measurements [3]. Therefore, can be said that several possible equatorial and polar diameters in a same piece of fruit.

According to [20], shape features can be measured independently (for example, by Fourier descriptors of the planar image boundary, invariant moments) or by combining size measurements (for example, circularity, aspect ratio, compactness, eccentricity, roundness). Hence, the determination of fruit size

parameters allows simple shape sorting.

A new method had proposed in [21] for recognization of fruit shape using concepts of attractor and fractal dimension. Neural network was used for quantitative measure and evaluation of the irregularity (or regularity) of the fruit shape. Proposed method is more reliable and more sophisticated for automated classification.

The system proposed in [22] can efficiently obtain the shape characteristic by drawing the lines and then class with Kmeans clustering method for the strawberry image. The proposed automated strawberry grading system uses $\mathrm{L}^{*} \mathrm{a} \mathrm{b}^{*}$ color model. The size of fruit is decided based on the largest fruit diameter and color of strawberry is extracted using Dominant color method on $\mathrm{a}^{*}$ channel. The results show that

the strawberry size detection error is not more than 5\%, the colour grading accuracy is $88.8 \%$, and the shape classification accuracy is above $90 \%$. The average time to grade one strawberry is below $3 \mathrm{~s}$.

\section{COLOR FEATURE EXTRACTION AND ALGORITHMS}

The most essential character of natural image is color and texture, which plays a crucial role in visual perception. Color feature is mainly used to simplify a monochrome dilemma by improving separation or contrast. The progression of color classification involves extraction of useful information with reference to the spectral properties of object surfaces and discovering the best match from a set of known descriptions or class models to execute the recognition task[15].

In[4] , an intellectual fruit sorting system is explained which is based on color factor. In this method initially fruit area is segmented from an image using OHTA color space and blob extraction is then applied to identify fruit contour and finally then color ratio was calculated using HIS color space that acts as a classification feature followed by the bayes classifier applied for grading. Average accuracy for classification is $90 \%$ and performance of proposed system is unwavering and satisfying for apples.
A fruit recognization system was developed by S.Arivazhagan, R.Newlin Shebiah, S.Selva

Nidhyanandhan, L.Ganesan [5] based on color and texture. Various parameters are taken into consideration for grading of fruit like intensity, color, shape and texture. To finish it with minimum distance classifier is used for classification of fruit based on Wavelet transformed sub bands. Accuracy achieved was $91.06 \%$

To grade San-Fuji apples, neural network plays significant role to classify apples into five quality classes [23]. Pixels are obtained from image based on color and are given to the neural network as an input. Mean color of fruit is obtained from fruit image and grading is done based on fruit mean color, its variability and availability of diseased pixels and ratio of red color of fruit image. Accuracy is obtained up to

95\% based on global classification rate $(70 \%)$.

In [6] a new fruit recognization system has been proposed in which among the background image the area of the fruit region was debarred to extract the correct color feature values of the fruit. Mean of colour was calculated from RGB component. After calculating color of fruit, shape is measured by threshold segmentation which included geometrical properties like Area, perimeter, diameter, etc of fruit, size is calculated and KNN was applied for classification. Recognition rate achieved was near about $90 \%$.

J. Blasco, N. Aleixos, J. Gómez, and E. Moltó in [7] examined comparative analysis of five five different color spaces RGB, XYZ, HSI, La*b* and $\mathrm{Lu}^{*} \mathrm{v}^{*}$ for sorting of citrus fruits. To obtain classification functions in each color space Linear discriminant analysis was carried out using color coordinates. Using HIS color space $87 \%$ accuracy was achieved which was considered as highest classification.

A new system was developed in [8] to identify normal and five common diseased peel conditions namely canker, copper burn, greasy spot, melanose, and wind scar that acquired RGB images from ruby red grapefruits. For this experiment 39 images as samples were used. Using the color co-occurrence method for each fruit sample Texture features were determined from the transformed hue $(\mathrm{H})$, saturation $(\mathrm{S})$, and intensity (I) or using region-of interest images. On the basis of stepwise discriminant analysis and 14, 9 and 11 texture features were selected for three color combinations of HSI, HS, and I, respectively for selecting useful texture features for the said purpose different algorithms were developed. Classification models were constructed using the reduced texture feature sets through a discriminant function based on a measure of the generalized squared distance. The best classification accuracy of $96.7 \%$ was achieved by the model using 14 selected HSI texture features. Average classification accuracy and standard deviation were $96.0 \%$ and $2.3 \%$, respectively. For classifying new fruit samples the proposed mode is robust.

For automatic color grading of fruit direct color mapping technique is used in [9]. Preselected colors are used in the proposed method to calculate a unique set of coefficients for color space conversion. The three-dimensional RGB color space is converted into a small set of color indices unique to the

application. Date surface detection and date maturity evaluation of tomato are also evaluated which is a simple but effective method which can be applied to a variety of color 
grading applications that requires easy setting and adjustment of color preferences and achieved $95 \%$ of accuracy.

The algorithm successfully estimate lemon based on color and size in [10], the algorithm successfully identified lemon based on color and size using the RGB value of image and HIS value was calculated from RGB. Color of fruit is determined by average value of hue component and stored in database. Grading of lemon was resoluted with accuracy of $94.04 \%$ by comparing the information during sorting phase with available information stored inside database.

A new approach for grading pineapples based on color is presented in [19].Images of pineapples were collected and background was removed. RGB component was extracted from pineapple image. During the sorting stage, the color values obtained are saved in a database for training the Neural Network (NN). The test results show the highest accuracy achieved for grading pineapples is $75 \%$.

Table 1. Summary Of Different Fruits And Their Parameters

\begin{tabular}{|l|l|l|l|}
\hline Type of Fruit & Parameter & Accuracy & Reference \\
\hline Apple & Color & $90 \%$ & Guo Feng, 2004 \\
\hline Fruit $*$ & $\begin{array}{l}\text { Intensity } \\
\text { Color } \\
\text { Shape } \\
\text { Texture }\end{array}$ & $91.06 \%$ & $\begin{array}{l}\text { S.Arivazhagan, R.Newlin } \\
\text { Shebiah, 2010 }\end{array}$ \\
\hline Fruit* & $\begin{array}{l}\text { Area } \\
\text { Perimeter } \\
\text { Diameter } \\
\text { Color }\end{array}$ & $90 \%$ & Woo Chaw Seng, 2009 \\
\hline Fruit* & Color & $87 \%$ & \\
\hline Fruit* & Texture & $96.7 \%$ & J. Blasco, 2007 \\
\hline Tomatoes & Color & $95 \%$ & Dae Gwan Kim, 2009 \\
\hline Lemon & $\begin{array}{l}\text { Color } \\
\text { Size }\end{array}$ & $94.04 \%$ & Mah- Kye Lee, 2011 \\
\hline Pineapple & Color & $75 \%$ & J. I. Asnor,2013nazhand, 2010 \\
\hline
\end{tabular}

*: Generalized algorithm for all fruits

\section{SIZE FEATURE EXTRACTION AND ALGORITHMS}

Different electronic systems have been developed for nondestructive determination of horticultural producing dimensional size, over past few decades. According to the principle of measurement they are classified into six different groups.

Based on measurement of the volume of the gap between the fruit and the outer casing of embracing gauge equipment.

Systems that calculate fruit size by measuring the distance between a radiation source and the fruit contour, where this distance is computed from the time of flight (TOF) of the propagated waves.

Systems that rely on the obstruction of light barriers or blockade of light.

Two-dimensional (2-D) machine vision systems.

Three-dimensional (3-D) machine vision systems.

Other systems which includes systems based on internal images, such as computed tomography (CT) or magnetic resonance imaging (MRI), as well as some other approaches not

included in the other groups.

In [11] optical ring sensor system is developed. This lightblocking-based system consists of a circular frame on which a large number of infrared transmit- ters and receivers are alternately arranged. Every receiver detects the light emitted by the activated transmitter owing to the Lambertian response of all the transducers when the inner space of the ring is clear. The receivers obscured from the activated transmitter as soon as an object introduced in the ring, a shadow zone appears. The transmitters

are switched on in sequence round the ring, with the emitted light rays representing chords in the circumference.To approximate the object's contour the two closest noninterrupted chords, or 'tangential' chords, are used. For 2D and 3D objects the same algorithms are used. The difference between the area of the whole circle encompassed by the ring and the sum of the triangles and segments of the circle that comprise the gap between the object's contour and the ring computes thr fruit cross-sectional area.

$$
A_{\mathrm{f}}=\pi r^{2}-\sum\left(A_{\mathrm{T}}+A_{\mathrm{C}}\right)
$$

To determine fruit size a technique can be used is time of flight(TOF) laser range finding. Besl P.J. in [12] compared two techniques used in laser range finders, which are commonly known as laser scanners: TOF and triangulation. Among which triangulation is one of the most accurate methods for reconstructing the 3D surface of an object, whereas TOF laser range finders measures the distance from the scanner to a surface by timing the round-trip time of a pulse of light. TOF laser finders are better suited to measurement with a large depth of field even in order of kilometers compared to triangulation laser range finders, 
therefore they are not usually used for industrial inspection. Their accuracy is about $10-4 \mathrm{~m}$, whereas triangulation laser range finders have an accuracy of 10-6 m.

Chalidabhongse in [13] used the VI method to classify mangoes in three size classes. They used four silhouettes, each of which was obtained with one of four cameras. The volume was estimated by counting the number of voxels belonging to the fruit using the known calibration grid size.They obtained CVs of $7.3 \%, 5.5 \%$, and $13.1 \%$ with the reference laboratory instruments and $7.9 \%, 4.3 \%$, and $14.6 \%$ with the vision system for the smallest, intermediate and largest mangoes, respectively. A conclusion was made by them that even though the systems gave bigger variations than the laboratory reference instrument, it was more efficient in terms of speed and time.

Hongshe Dang, Jinguo Song, Qin Guo presented algorithm [24] for Fruit Size Detection and Grading System. Embedded grading system is used to implement the algorithm. Advantages of the algorithm are high accuracy of grading, high speed and low cost.

In [25], Quality grading of Coffee-Excelso Beans have been done using color, shape and size features that help us to find the green bean quality from a small sample.

Using a mechanical or an electronic weight sizer fruit weight direct determination can be performed. The main point of difference among both the concept is that that the mechanical sizer weight measurements are performed at the ejection points; each mechanical sizer ejection point represents a weight measurement point, whereas electronic weight sizers have a single weight measurement point per lane, typically arranged a short distance before the first ejection point.According to Studman in [14] the advantages of electronic weight sizers over mechanical sizers are: it is easier to recalibrate the machine for different size groups because it is not essential to adjust all the ejection points in isolation; the electronic weight grader can attain a more accurate weight measurement; and higher operating speeds are possible.

\section{CONCLUSION}

The literature review revealed that on fruit grading system using image processing lot of research has been carried out. Labor work for inspection of fruit grading can be replaced by image processing systems. India lacks of the scientific grading systems for the fruits. Despite the fact that India is having such a high rank in production of fruits and vegetables, they still do suffer from quality issues. If automatic grading systems are made available to them then it would save the time as well as the manual labor which will also enhance their quality issues related to the fruits or vegetables. The major problem for tackling with complex task is inclusion of knowledge in automatic grading system. The features for the grading of fruits are size, shape and color.

In this paper some of the techniques for all the three basic features that is size, color and shape are reviewed which helps us to understand different techniques with their point of accuracy. As India is negated of such automatic grading systems, development of such grading systems can economically help the planned agriculture. Different size, shape and color feature extraction techniques can be used for different kinds of fruits and vegetables. Every technique has their own advantages and disadvantages. Some methods are at more advanced stage than others because each method is based on estimation of feature parameters. But to make it a complete grading system advantages of different techniques can be combined together to improve the accuracy and efficiency of grading based on features. On top of it, new algorithms can be formed for the same grading task

Correlation between featured parameter and quality factor measurements enhance the effectiveness of the method. Initially back years ago grading method based on color did not acquire high speed electronic technologies and their sorting speeds were highly dependent on how well the measured parameters correlate with their quality factors. The combination of new imaging acquisition and high-speed

image processing techniques has provided new direction for researchers to develop many new and improved techniques for grading and sorting of fruits.

\section{REFERENCES}

[1] Hatou, K., Morimoto, T., De Jager, J., Hashimoto, Y., 1996. Measurement and recognition of 3-D body in intelligent plant factory. In: Abstracts of the International Conference on Agricultural Engineering (AgEng). Madrid, vol. 2, pp. 861-862 (Paper No. 96F-027)

[2] Aggarwal, J.K., Chien, C.H., 1989. Chapter 2: 3-D structures from 2-D images. In: Sanz, J.L.C. (Ed.), Advances in Machine Vision. Springer-Verlag Inc., New York,NY, pp. 64-121

[3] Affeldt Jr., H.A., Brown, G.K., Brusewitz, G.H., Delwiche, M.J., Hetzroni, A., Kranzler, G.A., et al., 1994. Dimension, shape and surface texture measurement on agricultural commodities. In: Proceedings of the BARD International Workshop on Nondestructive Technologies for Quality Evaluation of Fruits and Vegetables. ASAE, St. Joseph, Mich., USA, pp. 50-62.

[4] Guo Feng and Cao Qixin," Study on Color Image Processing Based Intelligent Fruit Sorting System",Proceedings of the 5" World Congress on Intelligent Control and Automation, pp. 4802-4805, June $15-19,2004$

[5] S.Arivazhagan, R.Newlin Shebiah, S.Selva Nidhyanandhan, L.Ganesan," Fruit Recognition usingColor and Texture Features", Journal of Emerging Trends in Computing and Information Sciences, VOL. 1, NO. 2, pp. 90-94, Oct 2010

[6] Woo Chaw Seng and Seyed Hadi Mirisaee, "A New Method for Fruits Recognition System," Electrical Engineering and Informatics, vol. 01, pp. 130-134, August 2009

[7] J. Blasco, N. Aleixos, J. Gómez, and E. Moltó, "Citrus sorting by identification of the most common defects using multispectral computer vision," Journal of Food Engineering, vol. 83, no. 3, pp. 384-393, December 2007

[8] Dae Gwan Kim, Thomas F. Burks, Jianwei Qin, Duke M. Bulanon," Classification of grapefruit peel diseases using color texture feature analysis", International Journal of Agricultural and Biological Engineering ,Vol. 2, No.3,pp. 41-50,September,2009.

[9] Dah-Jye Lee, James K. Archibald, and Guangming Xiong, "Rapid Color Grading for Fruit Quality Evaluation Using Direct Color Mapping," IEEE TRANSACTIONS ON AUTOMATION SCIENCE AND ENGINEERING, vol. 8, no. 2, pp. 292302,November 2011 
[10] M. Khojastehnazhand, M. Omid, and A. Tabatabaeefar, "Development of a lemon sorting system based on color and size," African Journal of Plant Science, vol. 4(4), pp. 122-127, April 2010

[11] Gall, H., 1997. A ring sensor system using a modified polar coordinate system to describe the shape of irregular objects. Measurement Science and Technology 8 (11), $1228-1235$

[12] Besl, P.J., 1989. Chapter 1: active optical range imaging sensors. In: Sanz, J.L.C. (Ed.), Advances in Machine Vision. Springer-Verlag Inc., New York, NY, pp. 1-63

[13] Chalidabhongse, T., Yimyam, P., Sirisomboon, P., 2006. 2-D/3-D vision-based mango's feature extraction and sorting. In: 9th International Conference on Control, Automation, Robotics and Vision (ICARCV'06), p. 6.

[14] Studman, C.J., 1999. Chapter 3: fruits and vegetables, section 3.3: handling systems and packaging. In: CIGR Handbook of Agricultural Engineering. vol. IV. ASAE, St. Joseph, Mich., USA, pp. 291-339.

[15] S.Arivazhagan, R.Newlin Shebiah, S.Selva Nidhyanandhan, , L.Ganesan; Fruit Recognition using Color and Texture Features, Journal of Emerging Trends in Computing and Information Sciences, vol. 1, no. 2, October 2010; pp. 90-94.

[16] Agriculture Economics and Importance of Agriculture in National Economy website [Online]

[17] http://agriinfo.in/?page=topic \&superid=9\&topicid=185.

[18] The Economic Survey 2012-13, Agricultural and ProcessedFood Products Export Development Authority (APEDA) The Union Budget 2013-14, Press Releases, Media Reports website [Online]

[19] http://www.ibef.org/industry/agriculture-india.aspx
[20] Sargent, S.A., Brecht, J.K., Talbot, M.T., Miller, W.M., Chau, K.V., 1988. Performance of

[21] packing line sizers for fresh market tomatoes. ASAE Paper No. 886590. St. Joseph, Mich., ASAE.

[22] J. I. Asnor, S. Rosnah, Z. W. H. Wan, and H. A. B. Badrul," Pineapple Maturity Recognition Using RGB Extraction",World Academy of Science,Engineering and Technology,vol.78 ,pp. 147-150,2013.

[23] Du, C.-J., Sun, D.-W., 2004. Recent developments in the applications of image processsing techniques for food quality evaluation. Trends in Food Science and Technology 15 (5), 230-249.

[24] T.Morimoto,T.Takeuchi,H.Miyata,Y.Hashimoto,"Pattern recognition of fruit shape based on

[25] the concept of chaos and neural networks",Computers and Electronics in Agriculture, vol. 26, pp. 171- 186, 2000

[26] Xu Liming and Zhao Yanchao, "Automated strawberry grading system based on image processing," Computers and Electronics in Agriculture, vol. 71, no. Supplement 1, pp. S32-S39, April 2010.

[27] Kazuhiro Nakano,"Application of neural networks to the color grading of apples", Computers and Electronics in Agriculture, Elsevier, pp. 105-116, 1997.

[28] Hongshe Dang, Jinguo Song, Qin Guo, "A Fruit Size Detecting and Grading System Based on Image Processing", 2010 Second International Conference on Intelligent Human-Machine Systems and Cybernetics, vol. 2, pp. 83-86, August 2010.

[29] Eduardo Carrillo and Alexander Aristizabal Penaloza, "Artificial vision to assure coffee-Excelso beans quality," in EATIS, Czech Republic, pp. 35,2009 